\title{
Influence of an iron intervention on the zinc status of young adult New Zealand women with mild iron deficiency
}

\author{
Nicolas R. Prosser ${ }^{1}$, Anne-Louise M. Heath ${ }^{1}$, Sheila M. Williams ${ }^{2}$ and Rosalind S. Gibson ${ }^{1}$ \\ ${ }^{1}$ Department of Human Nutrition, University of Otago, Dunedin, New Zealand \\ ${ }^{2}$ Department of Social and Preventive Medicine, University of Otago, Dunedin, New Zealand
}

(Received 10 August 2009 - Revised 6 January 2010 - Accepted 26 January 2010 - First published online 26 April 2010)

Interventions to combat mild Fe deficiency in women of childbearing age may affect $\mathrm{Zn}$ nutriture. We used dietary and laboratory indices to assess change in Zn status during a 4-month partially blinded placebo-controlled Fe intervention in women with low Fe stores (serum ferritin $<20 \mu \mathrm{g} / \mathrm{l}$ and $\mathrm{Hb} \geq 120 \mathrm{~g} / \mathrm{l}$ ) from Dunedin, New Zealand. Subjects aged 18-40 years were randomly assigned to three groups: dietary advice (diet group; DG; $n$ 29), daily Fe supplement with meals (supplement group; SG; $n 23 ; 50 \mathrm{mg}$ Fe as amino acid chelate) and placebo (placebo group; PG, $n$ 26). A validated semi-quantitative FFQ (SFFQ) was administered at baseline, and at 4, 8 and 15 weeks; fasting morning blood samples were assayed for serum $\mathrm{Zn}$, alkaline phosphatase (ALP) and C-reactive protein at baseline, and at 4, 8, 12 and 16 weeks; hair $\mathrm{Zn}$ and taste detection thresholds by electrogustometry were measured at baseline and at 16 weeks. Intakes of flesh foods and vitamin $\mathrm{C}$ but not $\mathrm{Zn}$ or Fe increased, whereas phytate and phytate:Zn molar ratios decreased (all $P \leq 0.01$ ) in the DG compared with the PG and SG, based on three SFFQ. Serum Zn increased in both the DG and PG (adjusted, $P \leq 0.002$ ), so the between-group difference was not significant; the lack of a parallel rise in the SG was significant when compared with the PG $(P=0.02)$. ALP activity (but not hair $\mathrm{Zn}$ or taste acuity) followed a similar trend. In conclusion, $\mathrm{Zn}$ status was not improved compared with placebo by an Fe-based dietary intervention. However, a daily moderate-dose Fe supplement with meals appeared to lower $\mathrm{Zn}$ status in these young adult women.

Serum zinc: Iron-zinc interaction: Iron supplements: Dietary interventions: Randomised controlled trials

Fe deficiency, with or without anaemia, is prevalent in infants, young children and women of childbearing age worldwide. Interventions involving dietary modification and diversification, Fe-fortified foods, or Fe supplementation, are often recommended to improve the $\mathrm{Fe}$ status of these at-risk groups. Women of childbearing age are especially at risk because pregnancy entails a high demand for $\mathrm{Fe}^{(1)}$.

In most countries, the risk of inadequate intakes of $\mathrm{Zn}$ is likely to be comparable with that of $\mathrm{Fe}$ because $\mathrm{Zn}$ and $\mathrm{Fe}$ have a similar distribution in the food supply and are affected by many of the same dietary absorption modifiers ${ }^{(2,3)}$. Indeed, low Fe stores have been a risk factor for suboptimal $\mathrm{Zn}$ status in several studies ${ }^{(4-7)}$.

Dietary interventions designed to enhance the Fe status of omnivorous women will include advice to increase intakes of flesh foods and enhancers of non-haem Fe absorption, and decrease intakes of inhibitors of non-haem Fe absorption $^{(8)}$. Flesh foods are rich sources of both readily available $\mathrm{Fe}$ and $\mathrm{Zn}$; and phytate, found in unrefined cereals and legumes, is a potent inhibitor of both $\mathrm{Fe}$ and $\mathrm{Zn}$ absorption ${ }^{(9)}$. Hence, if these dietary strategies are adopted, improvements in $\mathrm{Zn}$ as well as Fe status may be achieved. In contrast, Fe supplementation may have an adverse effect on $\mathrm{Zn}$ nutriture as a result of competition for a common absorptive pathway between $\mathrm{Zn}$ and non-haem $\mathrm{Fe}^{(10)}$. Studies examining this relationship, however, have shown mixed results ${ }^{(11-12)}$.

$\mathrm{Zn}$ is essential for optimal health and pregnancy outcome $\mathrm{e}^{(13)}$ and hence is as important as Fe for women of childbearing age. Women who have been advised to take Fe supplements may also be at risk of $\mathrm{Zn}$ deficiency, making it essential to ensure that any treatment regimen for $\mathrm{Fe}$ does not cause or exacerbate a pre-existing $\mathrm{Zn}$ deficiency state.

The objectives of the present study were, first, to investigate the effect of a dietary intervention, designed to enhance both the intake and bioavailability of Fe over 16 weeks, on dietary and laboratory indices of the $\mathrm{Zn}$ status of young adult women with mild Fe deficiency from Dunedin, New Zealand, and, second, to examine whether a moderate-dose Fe supplement taken daily with meals has an adverse effect on their $\mathrm{Zn}$ status. Mild Fe deficiency was defined as serum ferritin $<20$ $\mu \mathrm{g} / \mathrm{l}$ and $\mathrm{Hb} \geq 120 \mathrm{~g} / \mathrm{l}$. Zn status was evaluated using dietary indices, serum and hair $\mathrm{Zn}$ concentrations, serum alkaline phosphatase (ALP) activity (a Zn-dependent enzyme) and taste detection thresholds ${ }^{(14,15)}$. Details of the efficacy of the interventions on the primary outcome (i.e. serum ferritin) in this randomised controlled trial have been published earlier ${ }^{(16)}$.

Abbreviations: ALP, alkaline phosphatase; CRP, C-reactive protein; DG, diet group; GEE, generalised estimating equation; OCA, oral contraceptive agent;

PG, placebo group; SFFQ, semi-quantitative FFQ; SG, supplement group.

* Corresponding author: Nicolas R. Prosser, fax +64 3479 7958, email nicolasprosser@gmail.com 


\section{Subjects and methods}

\section{Details of the participants and study design}

Briefly, eighty-eight women aged 18-40 years with low $\mathrm{Fe}$ stores were recruited (March 1997 to April 1998) for the study from the greater Dunedin area, New Zealand, via newspaper advertisements, brochures and posters. Of these women, fifty had previously participated in a cross-sectional study $^{(17,18)}$. Inclusion criteria were presence of mild $\mathrm{Fe}$ deficiency (serum ferritin $<20 \mu \mathrm{g} / \mathrm{l}$ and $\mathrm{Hb} \geq 120 \mathrm{~g} / \mathrm{l}$ in the absence of infection), and consumption of a non-vegan Western-style diet. Exclusion criteria included anaemia, pregnancy or lactation, and health problems (for example, eating disorders) or medication ${ }^{(19)}$ likely to influence biochemical $\mathrm{Fe}$ and $\mathrm{Zn}$ status. Participants were instructed to avoid: donating blood; changing their normal exercise levels; or taking dietary supplements containing $\mathrm{Fe}$ (except as supplied), $\mathrm{Zn}$, vitamin $\mathrm{C}$ or $\mathrm{Ca}$ during the study period. Adherence to the exclusion criteria was monitored via an intervieweradministered questionnaire at the monthly appointments.

Participants were randomised to groups balanced by block stratification for serum ferritin $(<12 \mu \mathrm{g} / \mathrm{l}$ or $12-19 \mu \mathrm{g} / \mathrm{l})$; amount of menstrual blood loss as described earlier ${ }^{(17,20)}$; and whether vegetarian or not. Each treatment group was (by dice) assigned a number which was concealed in envelopes and placed in blocks of three in randomly ordered boxes, one for each stratum. The research dietitian allocated the next envelope in the appropriate box to incoming participants, and was subsequently unblinded to the treatment designation. Blinding of all the other research staff was maintained until completion of the study. The trial profile detailing the numbers of participants recruited, then randomised, and withdrawals or exclusions from each experimental group, is given in Fig. 1.

Participants in the supplement group (SG; $n$ 23) and placebo group (PG; $n$ 26) received a daily tablet (Fe supplement or placebo, respectively) packaged in blister packs. The $\mathrm{Fe}$ supplement provided $50 \mathrm{mg}$ elemental $\mathrm{Fe}$ in the form of an amino acid chelate (bis-glycino Fe II: 'FerroChel'; Albion Laboratories Inc., Clearfield, UT, USA). The placebo tablets, of maltodextrin, were identical in appearance (dark-green opaque gel capsules) and packaging, and provided by the same company. Participants in the diet group (DG; $n$ 29) received placebo tablets and individual advice from the

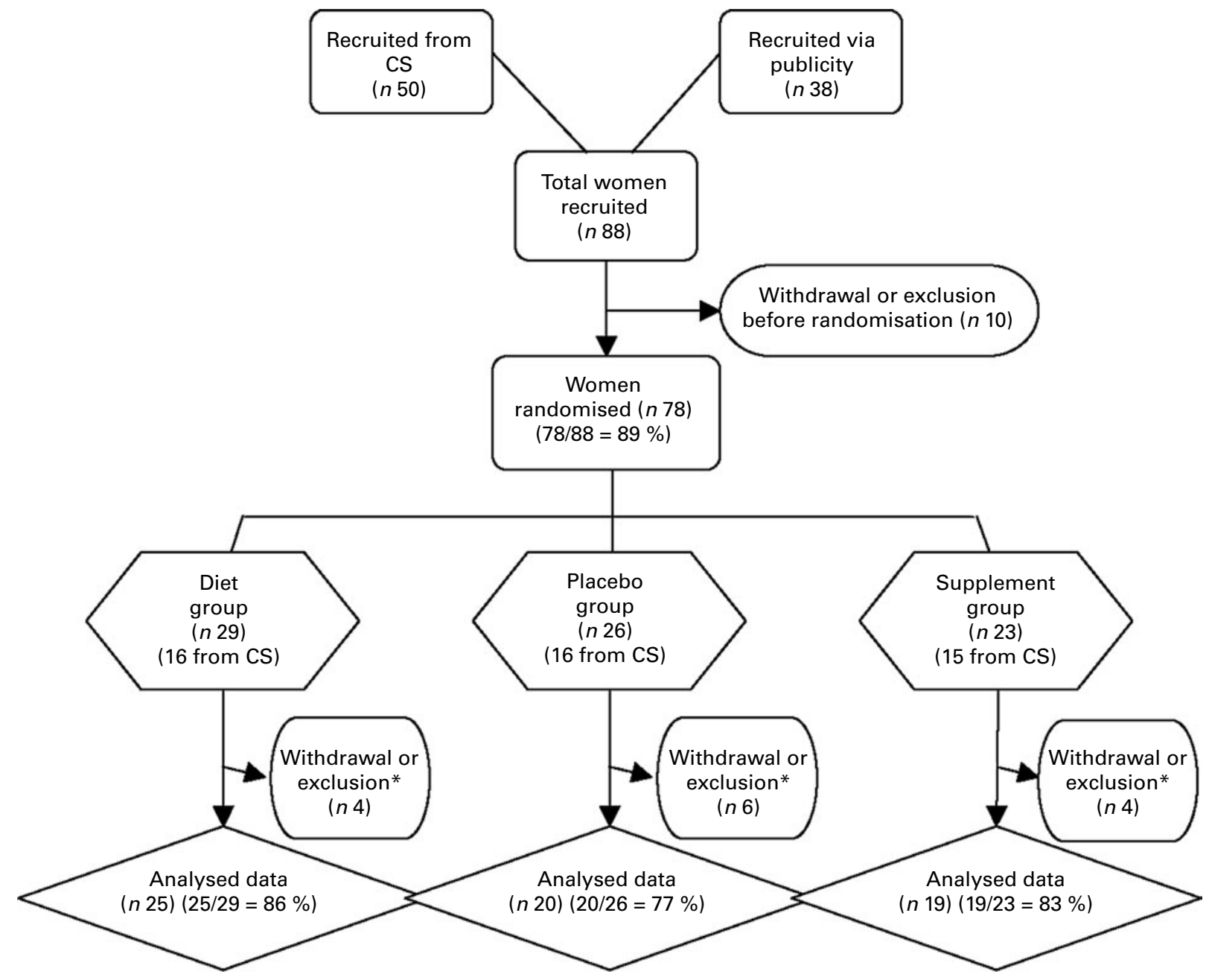

Fig. 1. Recruitment, withdrawals, exclusions and intervention group numbers. CS, cross-sectional phase of Women's Iron Study ${ }^{(17,18)}$. ${ }^{\star}$ There was one withdrawal from the diet group. There were four withdrawals from the placebo group and three from the supplement group. There were three exclusions from the diet group (two due to anaemia before the start of the intervention and one due to anaemia during the intervention), two from the placebo group (one due to anaemia before the start of the intervention and one due to anaemia during the intervention) and one from the supplement group (due to anaemia before the start of the intervention). 
dietitian based on a set of Fe-based dietary strategies given at baseline, and at weeks 2 and 6, with telephone check-ups at weeks 10 and $14^{(16)}$. Each DG participant was also given a cast-iron frying pan ${ }^{(21)}$, vitamin C-rich fruit juices (Frucor Beverages Ltd, Manukau City, New Zealand) and syrups (Barker's Fruit, Geraldine, New Zealand) to be consumed with meals, and recipe books and leaflets (New Zealand Pork, Wellington, New Zealand; Beef \& Lamb Marketing Bureau, Auckland, New Zealand). All participants were instructed to take one tablet per $d$ with a meal. Possible side effects were fully discussed and recorded daily by the participants on calendars, as were any other concerns that could have had a negative effect on adherence. Adherence to the tablets was assessed by both the calendar records and tablet counts, and to the dietary strategies by counselling sessions and telephone check-ups, with more formal assessments via a previously validated semi-quantitative FFQ (SFFQ) ${ }^{(22)}$. Of the tablets supplied, $97 \%$ were taken by the SG, with compliance in the PG and DG at 94 and $95 \%$, respectively.

At baseline, sociodemographic, general health and wellbeing data were collected via self-administered questionnaires, and participants were assisted to complete the computerised SFFQ. Selected anthropometric measures were taken ${ }^{(16)}$ together with scalp hair samples and fasting venepuncture blood samples using the International Zinc Nutrition Consultative Group (IZiNCG) procedures ${ }^{(23)}$. The SFFQ was administered again at 4, 8 and 15 weeks, and biochemical assays and anthropometry again at 4, 8, 12 and 16 weeks. Scalp hair samples and taste detection thresholds were taken at baseline and at the end of the intervention. Nutrient intakes from the three SFFQ were averaged for an estimate of intervention intake, and changes from the baseline SFFQ intakes were calculated.

The present study was conducted according to the guidelines laid down in the Declaration of Helsinki and all procedures involving human subjects were approved by the Southern Regional Health Authority Ethics Committee (Otago, New Zealand). Written informed consent was obtained from all participants.

\section{Laboratory methods}

Each participant's serum samples were analysed in one batch, with samples of pooled and/or reference material interspersed at approximately $10 \%$ frequency. Serum $\mathrm{Zn}$ analysis was performed by flame atomic absorption spectrophotometry (AAS) using a modification of the method of Smith et al. ${ }^{(24)}$. The percentage $\mathrm{CV}$ for $\mathrm{Zn}$ analyses from pooled serum samples was $6 \cdot 1 \%$ (mean value $12.6 \mu \mathrm{mol} / \mathrm{l} ; n$ 21). A certified reference material (CRM A-13, freeze-dried bovine blood; Analytical Quality Control Services, International Atomic Energy Agency, Vienna, Austria) and a reference human serum (normal range, U2A/product no. 66816; Utak Laboratories Inc., Valencia, CA, USA) were also analysed to assess the accuracy of the method. On a dry-weight basis, CRM A-13 produced a mean of 2.05 (SEM 0.02) $\mu \mathrm{mol} / \mathrm{g}$ (CV $4.6 \% ; n$ 23) compared with the certified value of 1.99 (95\% CI $1.84,2 \cdot 14) \mu \mathrm{mol} / \mathrm{g}$. The reference human serum yielded a mean of $15 \cdot 3(\operatorname{SEM} 0 \cdot 15) \mu \mathrm{mol} / \mathrm{l}(\mathrm{CV} 4.9 \% ; n 21) \mathrm{com}-$ pared with the assigned value of 13.8 (range $10 \cdot 7-16 \cdot 8) \mu \mathrm{mol} / \mathrm{l}$.

Scalp hair samples (approximately $100 \mathrm{mg}$ ) were washed using non-ionic detergent by a modification of the method of Harrison et al. ${ }^{(25)}$, now one of the standard methods recommended by the International Zinc Nutrition Consultative Group (IZiNCG) ${ }^{(23)}$, and then analysed by flame AAS following wet digestion of hair with $1.2 \mathrm{ml}$ of ultra-pure $\mathrm{HNO}_{3}$ (700 g/l) (AristaR; BDH Laboratory Supplies, Palmerston North, New Zealand). Analyses of samples ( $n$ 48) of a powdered pooled-hair sample gave a $\mathrm{CV}$ of $2.7 \%$ and mean of 3.40 (SEM 0.013) $\mu \mathrm{mol} / \mathrm{g}$. Serial analyses ( $n$ 33) of a certified reference material (Community Bureau of Reference RM no. 397; Institute of Reference Materials and Measurements, Geel, Belgium) yielded a mean value of 3.05 (SEM 0.018) $\mu \mathrm{mol} / \mathrm{g}$ (CV $3.4 \%$ ) compared with the certified value of 3.04 (SEM 0.08) $\mu \mathrm{mol} / \mathrm{g}$ (i.e. 199 (SEM 5) $\mu \mathrm{g} / \mathrm{g}$ ). Serum ALP was analysed on a Cobas-Mira Plus (Roche Diagnostics, Hoffmann-La Roche Ltd, Basel, Switzerland). Precision was assessed by replicate analyses of pooled serum (mean 49.9 (SEM 0.31 ) U/l; CV $3.5 \% ; n$ 31) and accuracy by analyses of certified reference materials in both a high (PrecipathU: mean 211 (SEM 0.61) U/l; CV 1.6\%; $n$ 30; manufacturer's target value 212 (SEM 13) U/l) and normal range (PrecinormU: mean 70.8 (SEM 0.55) U/l; CV $4.3 \% ; n$ 31; target value 68.4 (SEM 4.1) U/l). Hb, packed cell volume, serum C-reactive protein (CRP), ferritin and transferrin receptor were also analysed, as described elsewhere ${ }^{(16)}$.

Taste detection thresholds for 'electrogustometric' taste were measured at baseline and post-intervention by the same researcher using an electrogustometer (EGM; Campus Electronics, University of Otago, Dunedin, New Zealand). The electrogustometer was adapted from the design of Grant et $a{ }^{(26)}$ to permit measurement of threshold values as low as $1 \mu \mathrm{A}$. A threshold level was determined by alternately lowering and raising the current to determine the smallest stimulus that could be correctly discriminated with at least $75 \%$ accuracy. The technical error of measurement (TEM) based on electrogustometry assessments, repeated $3 \mathrm{~d}$ apart at the same time each morning on nine women aged 22-35 years, was $0.54 \mu \mathrm{A}$ (relative TEM $27 \%$ ).

\section{Statistical analysis}

Normality of distribution of values was assessed by the Kolmogorov-Smirnov and Shapiro-Wilk statistics, and, where there was inconsistency between these two, also by visual inspection of histograms with reference to measures of skew and kurtosis. Logarithmic transformations were used where appropriate to normalise data. Homogeneity of variance was ascertained for all statistical procedures using Levene's test.

Means (and 95\% CI) are reported for normally distributed data; for non-normally distributed data, medians (with interquartile range) or geometric means (for log-normal data) are reported, unless otherwise indicated. Treatment effects relative to placebo were estimated as changes across the five monthly measures for serum $\mathrm{Zn}$ and ALP, after adjustment for potential confounders, using generalised estimating equation (GEE) analysis. For the dietary intakes, hair $\mathrm{Zn}$ concentrations and taste acuity assessments, post-intervention values were adjusted for baseline values and compared by ANCOVA (unless otherwise indicated). Pairwise comparisons were conducted between groups using Bonferroni adjustment for multiple comparisons ${ }^{(27)}$. At least three time points are required for GEE 
Table 1. Baseline demographic and other characteristics of participants in each intervention group

\begin{tabular}{|c|c|c|c|c|c|c|}
\hline & \multicolumn{2}{|c|}{$\begin{array}{l}\text { Diet group } \\
\quad(n 25)\end{array}$} & \multicolumn{2}{|c|}{$\begin{array}{l}\text { Supplement } \\
\text { group } \\
(n 19)\end{array}$} & \multicolumn{2}{|c|}{$\begin{array}{l}\text { Placebo } \\
\text { group } \\
(n 20)\end{array}$} \\
\hline & $n$ & $\%$ & $n$ & $\%$ & $n$ & $\%$ \\
\hline \multicolumn{7}{|l|}{ Age (years) } \\
\hline Median & \multirow{2}{*}{\multicolumn{2}{|c|}{$\begin{array}{c}31 \cdot 5 \\
21 \cdot 8,38 \cdot 2\end{array}$}} & \multirow{2}{*}{\multicolumn{2}{|c|}{$\begin{array}{c}25 \cdot 0 \\
20 \cdot 0.35 .2\end{array}$}} & \multicolumn{2}{|c|}{23.9} \\
\hline 1st, 3rd quartiles & & & & & & 29.4 \\
\hline \multicolumn{7}{|l|}{ NZSEI class* } \\
\hline Median & \multirow{2}{*}{\multicolumn{2}{|c|}{$\begin{array}{c}3 \\
2,5\end{array}$}} & \multicolumn{2}{|c|}{3} & \multicolumn{2}{|c|}{2.5} \\
\hline 1st, 3rd quartiles & & & & & & \\
\hline \multicolumn{7}{|l|}{ BMI $\left(\mathrm{kg} / \mathrm{m}^{2}\right)$} \\
\hline Mean & \multirow{2}{*}{\multicolumn{2}{|c|}{23.9}} & \multicolumn{2}{|c|}{$22 \cdot 7$} & \multicolumn{2}{|c|}{$25 \cdot 1$} \\
\hline SD & & & \multicolumn{2}{|c|}{4.0} & \multicolumn{2}{|c|}{$5 \cdot 7$} \\
\hline Ethnicity: non-New Zealand European & 3 & 12 & 1 & $5 \cdot 3$ & 1 & 5 \\
\hline University degree & 8 & 32 & 6 & 32 & 8 & 40 \\
\hline Living with dependent children & 11 & 44 & 4 & 21 & 2 & 10 \\
\hline Negligible MPF intake $(<10 \mathrm{~g} / \mathrm{d})$ & 2 & 8 & 1 & $5 \cdot 3$ & 3 & 15 \\
\hline OCA use & 5 & 20 & 4 & 21 & 9 & 45 \\
\hline Blood drawn in afternoon & 4 & 16 & 0 & 0 & 1 & 5 \\
\hline $\mathrm{CRP} \geq 5 \mathrm{mg} / \mathrm{l}$ & 2 & 8 & 1 & 5 & 3 & 15 \\
\hline
\end{tabular}

MPF, meat, poultry and fish; OCA, oral contraceptive agent; CRP, C-reactive protein.

*New Zealand Socio-economic Index of Occupational Status ${ }^{(38)}$, classes 1 to 6 (1 representing the highest category), calculated as highest of self or partner, otherwise highest parent.

analysis, so this could only be conducted for serum $\mathrm{Zn}$ and ALP. Inter-relationships between indices were examined with Pearson's $(r)$ or Spearman's rank $(\rho)$ correlation coefficients. All probability values are for two-tailed tests with $P<0.05$ considered significant. Statistical procedures were carried out using SPSS version 11.0 (SPSS Inc., Chicago, IL, USA; http://www.spss.com), except for the GEE population-averaged procedures, which used STATA 10.0 (StataCorp LP, College Station, TX, USA; http://www.stata.com).

\section{Results}

\section{Baseline sociodemographic and health characteristics}

Table 1 displays baseline demographic and other characteristics for the three groups. Recruitment took place over a 14-month period (with no difference between intervention groups; Kruskal-Wallis $\left.\quad \chi^{2}(2)=0.71 ; \quad P=0.7\right)$; fifty-one participants $(80 \%)$ commenced the intervention between April and August inclusive (i.e. autumn/winter), with completion from late winter until early summer.

\section{Dietary intakes}

At baseline, there were no significant differences in the dietary intakes (median; 1st, 3rd quartiles), or the prevalence of $\mathrm{Zn}$ intakes below the estimated average requirement $(6.5 \mathrm{mg} / \mathrm{d})^{(28)}$ among the three groups (Table 2). One-quarter of all participants had diets with phytate:Zn molar ratios $\geq 15$. Table 3 details the dietary changes for the three groups from baseline values. In the DG, significant increases (analysis of covariance pairwise comparisons, with Bonferroni adjustment, $P<0 \cdot 05$ ) in intakes of meat, poultry and fish, and vitamin $C$, and significant decreases $(P<0.05)$ in intakes of phytate and phytate: $\mathrm{Zn}$

Table 2. Intakes of selected dietary variables by intervention group at baseline* (Medians and 1st and 3rd quartiles)

\begin{tabular}{|c|c|c|c|c|c|c|}
\hline & \multicolumn{2}{|c|}{ Diet group ( $n$ 25) } & \multicolumn{2}{|c|}{ Supplement group (n 19) } & \multicolumn{2}{|c|}{ Placebo group ( $n$ 20) } \\
\hline & Median & 1st, 3rd quartiles & Median & 1st, 3rd quartiles & Median & 1st, 3rd quartiles \\
\hline $\mathrm{Zn}(\mathrm{mg} / \mathrm{d})$ & $10 \cdot 5$ & $8.5,13.2$ & $10 \cdot 8$ & $9 \cdot 3,13 \cdot 8$ & $9 \cdot 3$ & $7.9,10.9$ \\
\hline Fibre (NSP) (g/d) & $20 \cdot 0$ & $16 \cdot 6,29 \cdot 5$ & $21 \cdot 0$ & $17 \cdot 1,25 \cdot 1$ & $18 \cdot 9$ & $10 \cdot 4,22 \cdot 6$ \\
\hline Phytate $(\mathrm{mg} / \mathrm{d})$ & 1207 & 622,1898 & 1122 & 787,1467 & 994 & 579,1274 \\
\hline PZR & 11.9 & $6 \cdot 5,16 \cdot 1$ & $10 \cdot 2$ & $7 \cdot 8,13.4$ & 11.5 & $8 \cdot 8,14.4$ \\
\hline \multicolumn{7}{|l|}{$P Z R \geq 15$} \\
\hline$n$ & & 8 & & 4 & & 4 \\
\hline$\%$ & & 32 & & 21 & & 20 \\
\hline MPF (g/d) & 118 & 68,146 & 140 & 101,162 & 95 & 55,130 \\
\hline Protein $(g / d)$ & 78 & 67,97 & 76 & 64,92 & 67 & 61,84 \\
\hline $\mathrm{Fe}(\mathrm{mg} / \mathrm{d})$ & $10 \cdot 2$ & $8 \cdot 3,13.9$ & $11 \cdot 2$ & $9.8,13.5$ & $9 \cdot 8$ & $8 \cdot 8,12.5$ \\
\hline $\mathrm{Ca}(\mathrm{mg} / \mathrm{d})$ & 795 & 629,962 & 583 & 521,880 & 791 & 498,993 \\
\hline Vitamin C $(\mathrm{mg} / \mathrm{d})$ & 105 & 60,138 & 98 & 73,135 & 107 & 53,155 \\
\hline
\end{tabular}

PZR, phytate:Zn molar ratio; MPF, meat, poultry and fish.

* Dietary energy not reported, as the semi-quantitative FFQ was not designed or validated for energy intake. 


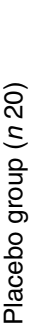

molar ratio occurred from baseline values in comparison with the changes in intakes observed in the PG and in the SG.

\section{Laboratory assessment}

Highly significant correlations $(P<0.0001)$ were noted for individual participants at each time period for serum $\mathrm{Zn}$ concentrations and for ALP activities. Pearson correlation coefficients ranged from $r 0.86$ to $r 0.92$ for serum $\mathrm{Zn}$ (mean intra-individual $\mathrm{CV} 3.0 \%$ ), and $r 0.87$ to $r 0.94$ for log-transformed ALP (mean CV 9.5\%).

Mean serum $\mathrm{Zn}$ and ALP values with their standard errors for the three groups at each time point are shown in Fig. 2. Note that the mean baseline serum $\mathrm{Zn}$ value for the SG was noticeably higher - a trend that continued throughout all the monthly assessments for the SG. Nevertheless, the difference in baseline serum $\mathrm{Zn}$ among the three groups was not significant $(P=0 \cdot 116)$ after adjustment for potential confounders (elevated CRP, oral contraceptive agent (OCA) use, afternoon blood sample and age). Baseline serum ALP activity did not differ significantly $(P=0.589)$ between groups. Results of the GEE analysis (Table 4) indicate that adjusted serum $\mathrm{Zn}$ values increased in the DG (adjusted, $P=0 \cdot 002$ ); however,
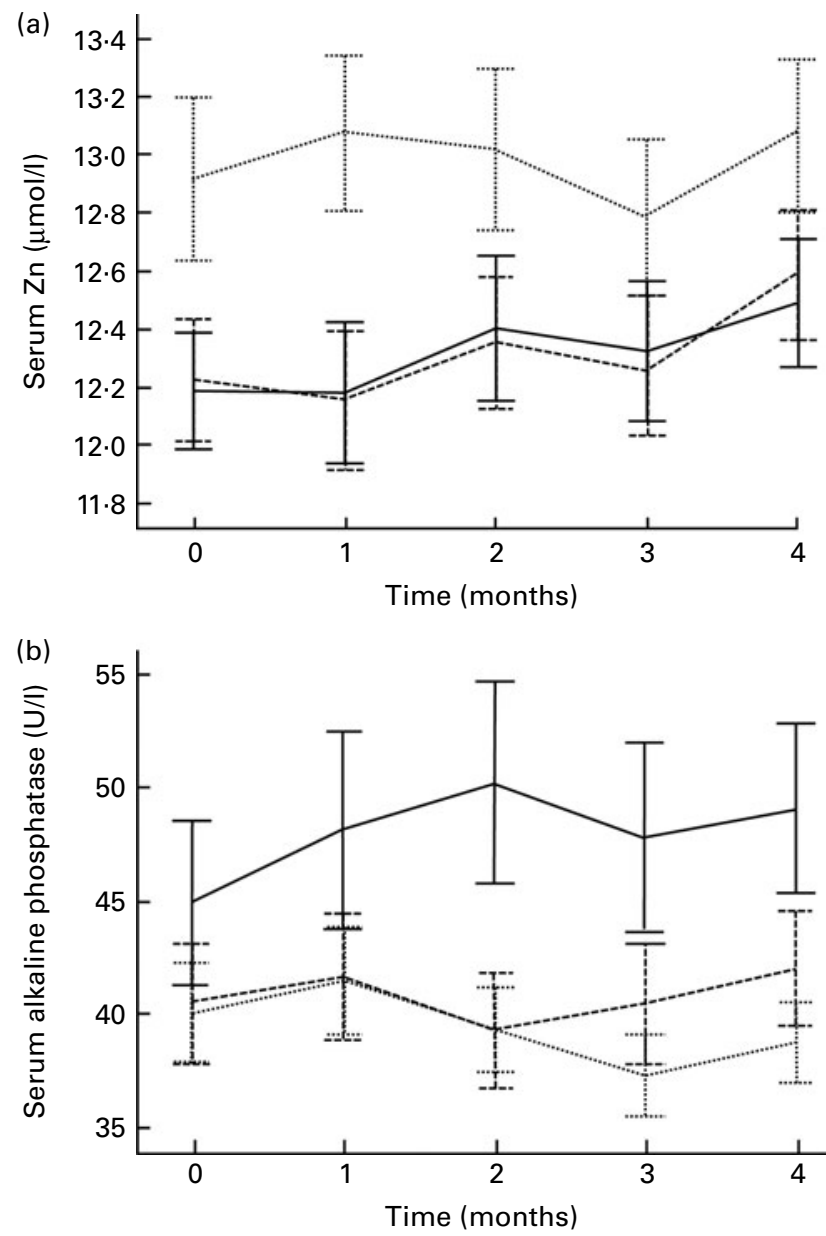

Fig. 2. Serum $\mathrm{Zn}(\mathrm{a})$ and alkaline phosphatase (b) values (not adjusted for confounders) for the three intervention groups, at baseline (time 0 ) and each month during the intervention. (---), Diet group; ( ..), supplement group; (-), placebo group. Values are means, with standard errors represented by vertical bars. 
Table 4. Generalised estimating equation $(\mathrm{GEE})^{\star}$ analysis of changes in serum zinc concentration and serum alkaline phosphatase (ALP) activity across the five monthly measures, for each intervention group, adjusted for confounders $†$

(Coefficients of slope and $95 \%$ confidence intervals)

\begin{tabular}{|c|c|c|c|}
\hline & Coefficient of slopeł & $95 \% \mathrm{Cl}$ & $P$ \\
\hline \multicolumn{4}{|l|}{ Serum Zn } \\
\hline $\mathrm{DG}(n 25)$ & 0.082 & $0.031,0.132$ & 0.002 \\
\hline $\mathrm{SG}(n 19)$ & -0.011 & $-0.070,0.047$ & 0.702 \\
\hline $\mathrm{PG}(n 20)$ & 0.074 & $0.030,0.119$ & 0.001 \\
\hline \multicolumn{4}{|c|}{ Difference between slopes: $\chi^{2}(2)=6.88(P=0.032)$} \\
\hline $\mathrm{DG} v \cdot \mathrm{PG}$ & 0.008 & $-0.060,0.076$ & 0.825 \\
\hline$S G v . P G$ & -0.086 & $-0.159,-0.012$ & 0.022 \\
\hline SG $v . D G$ & -0.093 & $-0.170,-0.017$ & 0.017 \\
\hline \multicolumn{4}{|l|}{ Serum ALP } \\
\hline $\mathrm{DG}$ & 0.173 & $-0.539,0.884$ & 0.634 \\
\hline SG & -0.543 & $-1.183,0.096$ & 0.096 \\
\hline$P G$ & 0.860 & $-0.311,2.031$ & 0.150 \\
\hline \multicolumn{4}{|c|}{ Difference between slopes: $\chi^{2}(2)=4.87(P=0.088)$} \\
\hline$D G v . P G$ & -0.687 & $-2.072,0.697$ & 0.331 \\
\hline$S G v . P G$ & -1.404 & $-2.757,-0.050$ & 0.042 \\
\hline$S G v . D G$ & -0.716 & $-1.674,0.242$ & 0.143 \\
\hline
\end{tabular}

DG, diet group; PG, placebo group; SG, supplement group.

*Population-averaged model; Wald statistic for model: serum $\mathrm{Zn} \chi^{2}(9)=29.9$ $(P<0.001)$; serum ALP $\chi^{2}(7)=21.4(P=0.003)$.

†Potential confounders entered as covariates: for serum $\mathrm{Zn}$, C-reactive protein $\geq 5 \mathrm{mg} / \mathrm{l}$, oral contraceptive use, blood sample drawn after noon (fasting status unknown), age; for serum ALP, oral contraceptive use, age.

$\ddagger$ Value of coefficient of slope represents: change in serum $\mathrm{Zn}(\mu \mathrm{mol} / \mathrm{l})$ per month; change in serum ALP (U/I) per month.

almost the same rise occurred in the PG, and the difference between the two groups was not significant. In contrast, a small decrease in adjusted serum $\mathrm{Zn}$ concentrations in the SG was significant when compared with the PG $(P=0.022)$. Similarly, adjusted serum ALP activity also tended to decline in the SG, with the change relative to the PG just showing statistical significance $(P=0 \cdot 042)$. There was a non-significant increase in adjusted ALP activity in both the PG and DG, and the difference between these was not significant (Table 4).

Hair $\mathrm{Zn}$ values were not significantly affected by concurrent infection (CRP $>5 \mathrm{mg} / \mathrm{l}$ ) or OCA use at baseline or post-intervention. Concentrations tended to be higher post-intervention in all three groups compared with baseline, with a correlation of $r 0.71(P<0.0001)$, but no significant inter-group differences existed (Table 5).

The median electrogustometric taste detection threshold values for each of the intervention groups at both baseline and post-intervention were identical $(3 \mu \mathrm{A})$, with only small differences in the quartiles. No group showed a significant change between baseline and post-intervention, and no inter-group differences were apparent (all $P>0 \cdot 6$ ).

\section{Inter-relationships among dietary and laboratory indices of zinc status}

There were no consistent significant correlations between the dietary variables and any of the laboratory indices, when expressed either as baseline or intervention values, or as change in both parameters during the intervention. The change in taste thresholds was negatively correlated with change in hair $\mathrm{Zn}$ concentrations ( $\rho-0.34 ; P=0 \cdot 011)$, whereas correlations between serum and hair $\mathrm{Zn}$ concentrations at start and post-intervention were positive $(P<0 \cdot 0001)$. Serum $\mathrm{Zn}$ and $\log$-ALP at the five sample times, excluding those with infection and OCA users, also tended to be weakly and positively correlated. The relationship between hair $\mathrm{Zn}$ values and ALP was less consistent.

Serum Zn concentration correlated positively with $\log$-transformed serum ferritin at baseline $(r 0.32 ; P=0.035$; $n 44)$ and post-intervention ( $r 0.43 ; P=0.005 ; n 41)$ (excluding potential confounders, i.e. OCA users and those with $\mathrm{CRP} \geq 5 \mathrm{mg} / \mathrm{l}$ ). Analysis by intervention group indicated some differences with the highest correlation being found post-intervention in the SG $(r 0 \cdot 59 ; P=0 \cdot 046 ; n$ 12). Positive correlations were also evident between serum ferritin and hair Zn concentrations, although only significant for the final hair samples when subjects with elevated CRP and OCA users were excluded $(r 0.36 ; P=0.022 ; n 40)$. There were no consistent relationships of serum ferritin with ALP values, nor with taste thresholds.

\section{Discussion}

The present study suggests that a dietary intervention that improves Fe status ${ }^{(16)}$ does not necessarily increase indices of $\mathrm{Zn}$ status. Indeed, serum $\mathrm{Zn}$ values rose significantly in both the DG and PG by the end of the study, a trend that was not observed in the SG. Instead, the small decrease in serum $\mathrm{Zn}$ concentrations in the SG, significant when compared with the PG $(P=0 \cdot 022)$, suggests that a daily Fe supplement of $50 \mathrm{mg} / \mathrm{d}$ taken with meals may compromise $\mathrm{Zn}$ status in women who are mildly $\mathrm{Fe}$ deficient. Interestingly, serum ALP activity also tended to decrease in the SG, a trend that was significant when compared with the PG $(P=0 \cdot 042)$.

Table 5. Hair zinc concentrations ( $\mu \mathrm{mol} / \mathrm{g}$ ) at baseline and post-intervention, change during the intervention, and comparisons between groups (Mean unadjusted values and $95 \%$ confidence intervals)

\begin{tabular}{|c|c|c|c|c|c|c|c|}
\hline & \multicolumn{2}{|c|}{ Diet group $(n 23)^{\star}$} & \multicolumn{2}{|c|}{ Supplement group $(n 17)^{*}$} & \multicolumn{2}{|c|}{ Placebo group $(n 17)^{\star}$} & \multirow[b]{2}{*}{$P$ between groups } \\
\hline & Mean & $95 \% \mathrm{Cl}$ & Mean & $95 \% \mathrm{Cl}$ & Mean & $95 \% \mathrm{Cl}$ & \\
\hline Baseline $(A)$ & $2 \cdot 81$ & $2 \cdot 66,2.96$ & $2 \cdot 79$ & $2 \cdot 61,2.98$ & 2.57 & $2 \cdot 41,2 \cdot 74$ & $0.053 \dagger$ \\
\hline Post-intervention (B) & $2 \cdot 81$ & $2.66,2.95$ & 2.85 & $2.71,2.99$ & 2.66 & $2 \cdot 51,2 \cdot 81$ & $0.111 \dagger$ \\
\hline Change $(B-A)$ & +0.001 & $-0.115,0.117$ & +0.058 & $-0.088,0.204$ & +0.087 & $-0.053,0.226$ & $0.601 \dagger$ \\
\hline Post-intervention adjusted for baseline & $2 \cdot 76$ & $2 \cdot 66,2 \cdot 86$ & $2 \cdot 81$ & $2.70,2.93$ & $2 \cdot 76$ & $2 \cdot 64,2 \cdot 87$ & $0.746 \ddagger$ \\
\hline
\end{tabular}

*Data included for participants providing both hair samples. One participant's values (supplement group) excluded due to analytical error.

† Significance of between-groups ANOVA.

$\ddagger$ Significance of analysis of covariance on intervention values with baseline as covariate. 
Our failure to observe a positive effect of the dietary intervention on $\mathrm{Zn}$ nutriture in these women is probably associated with several factors. For instance, the dietary intervention did not increase dietary $\mathrm{Zn}$ intake, although the increase in intakes of meat, poultry and fish ( $25 \mathrm{~g}$ per d) and decrease in the phytate:Zn molar ratio (by 3 units) may have improved $\mathrm{Zn}$ bioavailability ${ }^{(9)}$, unless counteracted by a possible inhibitory effect of contaminant Fe from the cast-iron cookware ${ }^{(10)}$. Lack of sensitivity of the laboratory indices of $\mathrm{Zn}$ status employed may also be implicated, especially because only $6 \%(n 4)$ of the participants had baseline serum $\mathrm{Zn}$ concentrations said to be indicative of $\mathrm{Zn}$ deficiency ${ }^{(23)}$. Finally, our dietary intervention (i.e. 16 weeks) may have been too short to produce a detectable difference in serum $\mathrm{Zn}$ concentrations.

It is possible that the $\mathrm{Fe}$ supplement may have adversely affected either the absorption or metabolism of $\mathrm{Zn}$ as noted earlier, even though the dose $(50 \mathrm{mg})$ given was much less than that often prescribed to pregnant or anaemic women. Some intervention studies have reported an adverse effect of Fe supplements on $\mathrm{Zn}$ status in children ${ }^{(29)}$, although a recent review concluded that even if there is a negative effect, it is small ${ }^{(12)}$. These inconsistencies have led to the suggestion that both the Fe: $\mathrm{Zn}$ ratio $(\geq 2: 1)$ and the total amount of ionic species $(>25 \mathrm{mg})$ could determine whether a measurable effect on human $\mathrm{Zn}$ nutriture occurs ${ }^{(30,31)}$. In the present study, few participants had dietary ratios of Fe: $\mathrm{Zn} \geq 2: 1$, but one-quarter had intakes of $\mathrm{Zn}$ and $\mathrm{Fe}$ combined greater than $25 \mathrm{mg}$. Clearly, with the addition of the Fe supplement, both the ratio and the total ionic species exceeded these values for the SG participants. Some data suggest that such an effect might only occur when supplements are taken with water rather than with food ${ }^{(9,23)}$ because the dietary ligands released during digestion of food chelate the $\mathrm{Fe}$ and $\mathrm{Zn}$ and assist in their absorption. Interestingly, in the present study, participants were instructed to take their supplement (or placebo) with meals. We recognise that monitoring compliance to such a protocol is difficult. However, because our participants were strongly motivated, judging by their high level of adherence to the tablets, they were probably likely to conform to our study protocols.

The tendency for the serum $\mathrm{Zn}$ values to increase in our PG cannot easily be explained, and, to our knowledge, has not been reported previously. Studies investigating intravariability in serum or plasma $\mathrm{Zn}$ concentrations have reported $\mathrm{CV}^{(32,33)}$ without noting whether any time trends were apparent. The small dietary changes observed in the PG were unlikely to have such a significant effect on serum $\mathrm{Zn}$ values, and there is no evidence that the placebo tablet (maltodextrin) affects $\mathrm{Zn}$ absorption. Further, given that the Fe indices in the PG, unlike serum $\mathrm{Zn}$, did not exhibit any time trends ${ }^{(16)}$, the rise in serum $\mathrm{Zn}$ concentrations in the PG was probably not associated with the exclusion or withdrawal of participants from the study. The increasing values were also unlikely to be associated with length of time over which the serum samples were frozen because $\mathrm{Zn}$ is stable in frozen serum for prolonged periods ${ }^{(23)}$. Likewise, seasonal variation in serum $\mathrm{Zn}$ concentration was probably not a contributing factor because this trend has not been reported in adults and was not evident in our data.

We adjusted the serum $\mathrm{Zn}$ concentrations statistically in all groups including the PG to account for the impact of currently accepted confounders of serum $\mathrm{Zn}$ such as OCA use and acute infection or inflammation ${ }^{(23)}$. It is possible, however, that as the participants became more familiar with the study procedures and personnel, they may have experienced a progressive decrease in anxiety and stress levels at the blood test. This might perhaps have resulted in a corresponding increase in the serum $\mathrm{Zn}$ values of the participants. A metaanalysis of studies investigating effects of acute mental stress tasks (in human subjects) on induction of IL-6 in serum or plasma has shown an overall effect ${ }^{(34)}$. Animal studies have indicated that hepatic IL-6 is linked to hypozincaemia ${ }^{(35)}$.

It is noteworthy that neither hair $\mathrm{Zn}$ nor taste acuity in the SG followed the same decreasing trend as the serum $\mathrm{Zn}$ and ALP values. Moreover, only a weak association between taste acuity and serum or hair $\mathrm{Zn}$ concentrations was noted here.

Failure to find associations between dietary and biochemical indices for $\mathrm{Zn}$ is not unexpected, especially in view of the inconsistent relationships noted by earlier investigators ${ }^{(14)}$. Moreover, our SFFQ was more appropriate for use in assessing change in dietary $\mathrm{Zn}$ intake (repeatability: $\rho$ 0.67), rather than as an absolute quantitative measure (validity against $11 \mathrm{~d}$ weighed diet record: $\rho$ 0.42; A-LM Heath, unpublished results). Our correlations between successive sets of data for serum and hair $\mathrm{Zn}$ and ALP were substantially higher than literature reports on intra-variability ${ }^{(32,33)}$, and may account for the stronger inter-relationships amongst biochemical $\mathrm{Zn}$ indices in our dataset than have been reported previously.

Use of the GEE population-averaged procedure ${ }^{(36,37)}$ in the present study permitted a more robust assessment of the effects of the intervention on serum $\mathrm{Zn}$ and ALP. These indices have high day-to-day within-subject variability, which is a particular concern in a study with a relatively small number of subjects. By using GEE we were able to minimise these effects by analysing multiple time points for each individual. An additional advantage of GEE is that it is relatively unaffected by randomly missing values ${ }^{(37)}$. Nevertheless, because the present study was powered to detect changes in the $\mathrm{Fe}$ indices ${ }^{(16)}$ and not $\mathrm{Zn}$, our sample size may have been too small, contributing to the null findings (i.e. no difference in $\mathrm{Zn}$ status between the DG and PG). Further, although the research assistants performing the outcome measurements were unaware of the group allocations, our dietary intervention group could not be blinded to their treatment assignment. Hence, we cannot exclude the possibility that the changes in $\mathrm{Zn}$ status for the DG were linked with changes in dietary intakes that arose in response to participation, rather than the intervention per se.

Finally, because our study participants were self-selected, with the presence of mild Fe deficiency as an inclusion criterion, we cannot extrapolate our findings to all young women of childbearing age in New Zealand. It is plausible that women with mild Fe deficiency will respond differently to Fe supplementation compared with those with adequate Fe status or those with Fe-deficiency anaemia.

In summary, the present results did not show a positive role for the Fe-based dietary intervention in improving laboratory indices of $\mathrm{Zn}$ status in the premenopausal women studied here. In contrast, we found a significant adverse impact of supplemental Fe on serum $\mathrm{Zn}$ concentrations, despite our 
use of a low dose of supplemental Fe $(50 \mathrm{mg})$, and the advice to take it with meals to reduce any negative effect. Whether the level of reduction in serum $\mathrm{Zn}$ concentration in the present study was associated with any adverse functional consequences is uncertain.

\section{Acknowledgements}

The authors gratefully acknowledge the contribution of the women participating in this research. The authors also thank: Sue O'Brien, research dietitian; Rebecca Meikle, research nurse; Margaret Waldron, research nurse; the research assistants; Steve Tiszavari, Karl Bailey and Michelle Harper for laboratory expertise.

A part of the study was supported by a Laurenson Award. The study was funded by the Health Research Council, New Zealand.

The authors' responsibilities were as follows: N. R. P. collected hair samples and electrogustometry data, participated in other data collection and laboratory work, and performed statistical analyses (except GEE); A.-L. M. H. assisted with design and coordinated implementation of the study, and contributed to critical revision of the manuscript; S. M. W. conducted the GEE analysis and provided guidance on interpretation of other statistical analyses; R. S. G. designed and secured funding for the study; N. R. P. and R. S. G. participated in interpretation, and writing of the manuscript. N. R. P. had full access to all of the data and takes responsibility for its integrity and the accuracy of the data analysis.

None of the authors had any financial or personal conflicts of interest.

\section{References}

1. World Health Organization \& Food and Agriculture Organization (2004) Vitamin and Mineral Requirements in Human Nutrition, 2nd ed. Geneva: World Health Organization.

2. Prasad AS (1998) Zinc in human health: an update. $J$ Trace Elem Exp Med 11, 63-87.

3. Hunt JR (2002) Moving towards a plant-based diet: are iron and zinc at risk? Nutr Rev 60, 127-134.

4. Prasad AS, Miale A, Farid Z, et al. (1963) Zinc metabolism in patients with the syndrome of iron deficiency anemia, hepatosplenomegaly, dwarfism, and hypogonadism. J Lab Clin Med 61, 537-549.

5. Yokoi K, Alcock NW \& Sandstead HH (1994) Iron and zinc nutriture of premenopausal women: associations of diet with serum ferritin and plasma zinc disappearance and of serum ferritin with plasma zinc and plasma zinc disappearance. $J$ Lab Clin Med 124, 852-861.

6. Gibson RS, Heath A-LM, Prosser NR, et al. (2000) Are young women with low iron stores at risk of zinc as well as iron deficiency? In Proceedings of the 10th Meeting of Trace Elements in Man and Animals, Evian, France, 2-7 May 1999, pp. 323-328 [AM Roussel, RA Anderson and AE Favier, editors]. New York: Kluwer Academic.

7. Yokoi K, Sandstead HH, Egger NG, et al. (2007) Association between zinc pool sizes and iron stores in premenopausal women without anaemia. Br J Nutr 98, 1214-1223.

8. Hunt JR (2005) Dietary and physiological factors that affect the absorption and bioavailability of iron. Int J Vitam Nutr Res $\mathbf{7 5}$, $375-384$.
9. Lönnerdal B (2000) Dietary factors influencing zinc absorption. J Nutr 30, Suppl., 1378S-1383S.

10. Kordas K \& Stoltzfus RJ (2004) New evidence of iron and zinc interplay at the enterocyte and neural tissues. J Nutr 134, $1295-1298$.

11. Allen L \& Shrimpton R (2005) The International Research on Infant Supplementation Study: implications for programs and further research. J Nutr 135, Suppl., 666S-669S.

12. Fischer Walker C, Kordas K, Stoltzfus RJ, et al. (2005) Interactive effects of iron and zinc on biochemical and functional outcomes in supplementation trials. Am J Clin Nutr 82, $5-12$.

13. King JC (2000) Determinants of maternal zinc status during pregnancy. Am J Clin Nutr 71, Suppl., S1334-S1343.

14. Gibson RS (2005) Principles of Nutritional Assessment, 2nd ed. New York: Oxford University Press.

15. Lowe NM, Fekete K \& Decsi T (2009) Methods of assessment of zinc status in humans: a systematic review. Am J Clin Nutr 89, Suppl., 2040S-2051S.

16. Heath A-LM, Skeaff CM, O'Brien SM, et al. (2001) Can dietary treatment of non-anaemic iron deficiency improve iron status? J Am Coll Nutr 20, 477-484.

17. Heath A-LM, Skeaff CM, Williams SM, et al. (2001) The role of blood loss and diet in the aetiology of mild iron deficiency in premenopausal adult New Zealand women. Public Health Nutrition 4, 197-206.

18. Gibson RS, Heath A-LM, Limbaga MLS, et al. (2001) Are changes in food consumption patterns associated with lower biochemical zinc status among women from Dunedin, New Zealand? Br J Nutr 86, 71-80.

19. Thomas JA (1995) Drug-nutrient interactions. Nutr Rev 53, 271-282.

20. Heath A-LM, Skeaff CM \& Gibson RS (1998) Validation of a questionnaire method for estimating extent of menstrual blood loss in young adult women. $J$ Trace Elem Med Biol 12, 231-235.

21. Prinsen Geerligs PD, Brabin BJ \& Omari AAA (2003) Food prepared in iron cooking pots as an intervention for reducing iron deficiency anaemia in developing countries: a systematic review. J Hum Nutr Dietet 16, 275-281.

22. Heath A-LM, Skeaff CM \& Gibson RS (2000) The relative validity of a computerized food frequency questionnaire for estimating intake of dietary iron and its absorption modifiers. Eur J Clin Nutr 54, 592-599.

23. IZiNCG (2004) Assessment of the risk of zinc deficiency in a population and options for its control. Food Nutr Bull 25, Suppl. 2, S94-S203.

24. Smith JC Jr, Butronovitz GP \& Purdy WC (1979) Direct measurement of zinc in plasma by atomic absorption spectrophotometry. Clin Chem 25, 1487-1491.

25. Harrison WW, Yursachek JP \& Benson CA (1969) The determination of trace elements in human hair by atomic absorption spectrophotometry. Clin Chim Acta 23, 83-91.

26. Grant R, Ferguson MM, Strang R, et al. (1987) Evoked taste thresholds in a normal population and the application of electrogustometry to trigeminal nerve disease. J Neurol Neurosurg Psychiatry 50, 12-21.

27. Field A (2005) Discovering Statistics using SPSS, 2nd ed. London: Sage Publications.

28. National Health and Medical Research Council (2006) Nutrient Reference Values for Australia and New Zealand. Canberra, ACT: Commonwealth of Australia.

29. Smuts CM, Lombard CJ, Benadé AJ, et al. (2005) Efficacy of a foodlet-based multiple micronutrient supplement for preventing growth faltering, anemia, and micronutrient deficiency of infants: the four country IRIS trial pooled data analysis. J Nutr 135, Suppl., 631S-638S. 
30. Solomons NW (1986) Competitive interactions of iron and zinc in the diet: consequences for human nutrition. J Nutr 116, 927-935.

31. Solomons NW \& Ruz M (1997) Zinc and iron interaction: concepts and perspectives in the developing world. Nutr Res 17, 177-185.

32. Fraser CG (1992) Biological variation in clinical chemistry. An update: collated data, 1988-1991. Arch Pathol Lab Med 116, 916-923.

33. Lux O \& Naidoo D (1995) The assessment of biological variation components of copper, zinc, and selenium. J Nutr Biochem 6, 43-47.

34. Steptoe A, Hamer M \& Chida Y (2007) The effects of acute psychological stress on circulating inflammatory factors in humans: a review and meta-analysis. Brain Behav Immun 21, 901-912.

35. Liuzzi JP, Lichten LA, Rivera S, et al. (2005) Interleukin-6 regulates the zinc transporter Zip14 in liver and contributes to the hypozincemia of the acute-phase response. PNAS 102, $6843-6848$

36. Liang K-Y \& Zeger SL (1986) Longitudinal data analysis using generalised linear models. Biometrika 73, 13-22.

37. Ziegler A, Kastner C \& Blettner M (1998) The generalised estimating equations: an annotated bibliography. Biom J 40, $115-139$.

38. Davis P, McLeod K, Ransom M, et al. (1997) The New Zealand Socioeconomic Index of Occupational Status (NZSEI). Wellington, New Zealand: Statistics New Zealand. 